\title{
INFLUENCERS Y MOMPRENEURS:UNA EXPLORACIÓN POR EL REPERTORIO DIGITAL DE LA MATERNIDAD
}

\author{
INFLUENCERS AND MOMPRENEURS: AN EXPLORATION OF THE DIGITAL REPERTOIRE OF MOTHERHOOD
}

\section{INFLUENCERS E MOMPRENEURS:UMA EXPLORAÇ̃̃ODO REPERTÓRIO DIGITAL DA MATERNIDADE}

\section{RESUMEN}

Este artículo indaga la configuración discursiva del modelo de la "mamá emprendedora" en artículos periodísticos de la prensa argentina y en cuentas de redes sociales cuyas usuarias se auto-inscriben en ese modelo. Nos proponemos, en primer lugar, caracterizar particularidades y valoraciones de las narrativas maternales en el espacio digital; y seguidamente observar los rasgos y significaciones que constituyen el modelo de la madre emprendedora como aquella que encuentra una resolución adecuada en torno a las dificultades para la conciliación entre trabajo productivo y reproductivo. Para eso, apelamos al análisis cultural cualitativo desde la tradición de la crítica ideológica (Richard, 2009; Thompson, 1991) que posibilita indagar en la configuración de sentidos sociales en relación con sus condiciones materiales de producción (Angenot, 2010). A partir de la selección de algunos ejemplos, interrogamos los alcances y los límites de las ideas que se entretejen en contenidos que exhiben a los espacios digitales como una apertura a la colectivización de la maternidad y alientan a replicar experiencias maternas como las de la madre emprendedora.

Palabras clave: Maternidad. Representaciones. Medios Sociales.

\begin{abstract}
This article explores the discursive configuration of the "entrepreneurial mom" model in newspaper articles in the Argentine press and in social media accounts whose users self-enroll in that model. We propose, first of all, to characterize particularities and evaluations of maternal narratives in the digital space; and then observe the traits and meanings that constitute the model of the entrepreneurial mother as one who finds an adequate resolution around the difficulties in reconciling productive and reproductive work. For this, we appeal to qualitative cultural analysis from the tradition of ideological criticism (Richard, 2009; Thompson, 1991) that makes it possible to investigate the configuration of social meanings in relation to their material conditions of production (Angenot, 2010). Based on the selection of some examples, we question the scope and limits of the ideas that are interwoven in contents that show digital spaces as an opening
\end{abstract}

Marina Sanchez de Bustamante

Universidad de Buenos Aires (UBA). Email: marina.sdb@gmail.com

Carolina Justo von Lurzer

Universidad de Buenos Aires (UBA). Email: justo.carolina@gmail.com 
to the collectivization of motherhood and encourage the replication of maternal experiences such as that of the entrepreneurial mother.

Keywords: Motherhood. Representations. Social Media.

\section{RESUMO}

Este artigo investiga a configuração discursiva do modelo da "mãe empreendedora" em artigos de jornais na imprensa argentina e em contas de redes sociais cujos usuários se auto-inscrevem nesse modelo. Propomos, primeiro, caracterizar particularidades e valorizações das narrativas maternas no espaço digital; e depois observar as características e significados que constituem o modelo da mãe empreendedora como alguém que encontra uma resolução adequada para as dificuldades de conciliar trabalho produtivo e reprodutivo. Para isso, apelamos à análise cultural qualitativa da tradição da crítica ideológica (Richard, 2009; Thompson, 1991) que nos permite investigar a configuração dos significados sociais em relação às suas condições materiais de produção (Angenot, 2010). A partir da seleção de alguns exemplos, questionamos o alcance e os limites das idéias que se entrelaçam em conteúdos que exibem espaços digitais como abertura para a coletivização da maternidade e incentivam a replicação de experiências maternas como as da mãe empreendedora.

Palavras-chave: Maternidade; Representações; Mídias Sociais.

\section{Introducción}

Este trabajo es un acercamiento exploratorio y reflexivo sobre un repertorio cultural de la maternidad que se ubica en plataformas de los medios sociales conectivos: las cuentas de "madres emprendedoras" en lo que Jose Van Dijck (2016) identifica como sitios de red social y sitios dedicados al contenido generado por usuarios. Nacidas inicialmente como blogs, actualmente la tendencia es que ese tipo de cuentas se multiplique en las redes sociales, por lo que los espacios periodísticos presentan este repertorio como "el fenómeno instamums ${ }^{1 " 2}$ y a sus propietarias bajo la clasificación de mamás bloggers 3 o influencers ${ }^{4}$ sobre maternidad5.

\footnotetext{
1 Anglicismo por acronimia entre Instagram y Mum (mamá, en inglés).

2 Queija, Ana Paula (17/8/2018). El fenómeno instamums: la maternidad de las influencers de moda. La Nación [online]. Recuperado de https://www.lanacion.com.ar/moda-y-belleza/el-fenomenoinstamums-maternidad-influencers-moda-nid2182603 (consulta: 25/11/2020).

3 Anglicismo que refiere a quien escribe un blog.

4 Anglicismo que refiere a personas que, por el alcance de su presencia en las redes sociales y por la credibilidad que se les asigna respecto a un tema, monetizan su capacidad de convocatoria a través alianzas comerciales con marcas y empresas y/o con la creación de productos propios.

5 S/A. Mami blogs: conocé las nuevas influencers (28/6/2018). Elle [online]. Recuperado de: https://elle. clarin.com/lifestyle/sociedad/mami-blogs-conoce-a-las-nuevas-influencers/ (consulta: 25/11/2020).
} 
Observamos que en la escena actual argentina las formas en que la maternidad se constituye en discurso social pueden ser escindidas en diferentes regímenes o repertorios culturales. En ese sentido, identificamos un primer conjunto constituido por la herencia de las mediatizaciones clásicas del siglo $\mathrm{XX}$, como los manuales de puericultura y las revistas dirigidas a las mujeres en general o a las madres en particular.

Ese repertorio, al que denominamos experto ${ }^{6}$, alimentó la naturalización y la normalización de muchas de las exigencias y atribuciones sociales a las mujeres (concibiéndolas exclusivamente como mujeres cis y heterosexuales). Es decir, la condensación de significados que ofrecieron los productos del repertorio experto y que, con reordenamientos semánticos aún ofrecen, se combinó en un conjunto de elementos estabilizados como propios de la maternidad "normal", o sea aquella comprendida colectivamente como la más lógica y la mejor.

Abonado con ímpetu, el arquetipo hegemónico de la maternidad de las mediatizaciones clásicas (exhibido, por ejemplo, en revistas "femeninas" y de "embarazo y cuidado infantil”, publicidades, manuales de puericultura y crianza y programas televisivos) difundió el imaginario de una madre biológica, heterosexual, recatada, consagrada a labores domésticas, a la crianza y al vínculo conyugal, tutelada por los saberes de las disciplinas médicas, pero guiada por el instinto y el amor como aspectos innatos de la "esencia femenina”. No obstante, en tiempos más recientes, tal idealización comenzó a ser perturbada.

Paulatinamente, en el siglo XXI el mercado de la cultura y los medios masivos produjeron nuevas narrativas que marcan un distanciamiento con el enfoque semántico heredado de las mediatizaciones clásicas. Esto no implica una inversión, pero sí una ampliación de los repertorios discursivos sobre la maternidad.

Por un lado, con algunas reconfiguraciones ligadas a las transformaciones históricas y culturales, continúa vigente el repertorio experto que estabilizó el imaginario de la maternidad como núcleo constitutivo de la feminidad. Los núcleos simbólicos más sedimentados de este repertorio circulan cotidianamente en diversos textos de la cultura de masas en los que la única emoción atribuida a las madres respecto al vínculo con sus hijos e hijas es la felicidad, en los que las madres son presentadas como responsables individuales y exclusivas del cuidado infantil, en los que el rol materno se despliega con alguna preocupación pero sin angustias y con absoluta eficiencia en su ejercicio, a partir de la incorporación de las consideraciones de las ciencias médicas y de otras disciplinas que producen saberes y profesionales sobre embarazo, maternidad y crianza.

Por el otro lado, las narrativas emergentes en el siglo XXI presentan modos de vivir que remiten a cambios atendibles en las subjetividades maternas. Identificamos a este repertorio discursivo como catártico porque los textos culturales en los que se manifiesta (literatura de ficción y no ficción, series televisivas, blogs, películas) dialogan explícita y críticamente con el repertorio experto. Estos productos

6 Identificamos a este repertorio como experto porque su modalidad enunciativa es explícitamente pedagógica -tiene el propósito de enseñar y aconsejar- y lleva la rúbrica de profesionales de la salud. 
interrogan, ridiculizan y denuncian distintas dimensiones del ideal hegemónico materno mediatizado y lo señalan reiteradamente como un verosímil en crisis en función de sus mandatos opresivos e inalcanzables.

De esta forma, el repertorio catártico problematiza la ideología maternalista y ofrece distintas tonalidades emocionales vividas por las madres (por ejemplo, el enojo, la tristeza, la frustración); además, diversifica los itinerarios vitales sobre la experiencia materna (las formas de constituir familia, la compatibilización entre el trabajo y la crianza, las negociaciones con los vínculos afectivos); y relativiza -con recursos como la comicidad y/o la ironía- la idea de la maternidad como fuente de dicha absoluta y como espacio simbólico de realización plena para las mujeres.

Pero además de estas dos zonas retóricas -que se amplía con una tercera centrada en las narrativas periodísticas sobre las biografías de las "mamis" de la farándula- las cuentas de madres influencers en los medios sociales emergen como otro conjunto de retóricas con características propias, tramadas al mismo tiempo con acentos de los demás repertorios.

Una particularidad de estas textualidades digitales asociadas a la experiencia materna es que los relatos que ponen a circular no se producen por empresas dedicadas a la producción de contenidos culturales. Sino que son producidas por usuarias que apelan a las lógicas de la socialidad y creatividad online a través de las plataformas que las corporaciones de internet ponen a disposición (Blogger, YouTube, Instagram, Twitter). No obstante, en muchos casos las llamadas influencers de la maternidad poseen conocimientos profesionales para la producción de contenidos que inscriben en el marco de un negocio, identificado como "emprendimiento", con el que obtienen ingresos monetarios. Tales ingresos se deben al vínculo comercial que mantienen con marcas que eligen a estas bloggers/instagramers para que promocionen productos, así como de la venta de servicios que ofrecen ellas mismas (por ejemplo, cursos y workshops para el desarrollo de emprendimientos comerciales).

Nos interesa aquí detenernos y recortar una zona de este voluminoso repertorio que nunca exploramos. En los diferentes regímenes de representación de la maternidad que venimos observando la tensión sobre el cruce entre trabajo productivo y trabajo reproductivo es una dimensión presente. Sin embargo, en esta zona discursiva esa tensión se expresa de modo acentuado, en de los contenidos que publican las usuarias, pero especialmente en notas y entrevistas periodísticas que comentan el fenómeno.

Nos interpela, en particular, que algunas de las propietarias de las cuentas -las que se divulgan por los medios masivos- se identifiquen a sí mismas o sean identificadas por el periodismo como "emprendedoras", una posición que se valora

7 Tanto el repertorio experto como el repertorio catártico escenifican centralmente madres cuya identidad de género remite a mujeres cis. Si bien el repertorio catártico amplía y complejiza la representación de modos de hacer familia en algunos de sus textos el giro retórico principal es la desidealización de la experiencia materna de mujeres cis. En tiempos recientes, una novela de la televisión abierta argentina que se emitió en horario prime time -Pequeña Victoria (Telefé, 2019)- incluyó una madre trans en el relato. 
por entenderse una estrategia que permite cumplir con las tareas de maternaje y simultáneamente desplegar una labor que genera ingresos económicos.

Ante este escenario nos interesa reflexionar sobre las implicancias ligadas a la configuración enunciativa de un modelo contemporáneo ofrecido en contenidos de los medios masivos, que se constituye a partir de la confluencia de discursos que lo alimentan en el contexto actual: el de la "mamá emprendedora". Para llevar adelante nuestra reflexión partimos de los siguientes interrogantes: ¿Cuál es el enlace entre trabajo productivo y trabajo reproductivo que presentan los medios masivos al constituir el modelo de la madre emprendedora? ¿Qué actitudes y acciones se valoran y se proponen como aspectos de la subjetividad materna?

\section{La Domesticidad Digital}

El crecimiento de los blogs sobre maternidad es un fenómeno global que se remonta por lo menos a 2010 (Visa Barbosa y Crespo Cabillo, 2015) mediatizado, como señalamos, por plataformas digitales a las que las usuarias apelaron para narrar sus experiencias y emociones en torno al ejercicio de maternar. La emergencia de estos relatos puede comprenderse como parte de los procesos de expresión pública de lo íntimo o puesta en escena de la extimidad (Sibilia, 2008) en articulación con la expansión de lo biográfico en tanto espacio donde la vida y la propia experiencia son núcleos de tematización que dan cuenta de una tonalidad propia de la subjetividad contemporánea (Arfuch, 2007).

Los acercamientos a producciones de la blogosfera materna señalan que proveen un lugar para la autoexpresión y la conexión con otras madres, así como de redefinición y lucha simbólica en torno a identidades y prácticas de maternaje (Orton-Johnson, 2017): es decir, las madres que escriben y participan en los blogs sobre maternidad acceden a un lugar de encuentro en el que discuten y expresan sus propias ideas y sentimientos sobre qué es ser madre y las maneras que eligen o sobrellevan el rol materno. En tal sentido, en las líneas narrativas de las bloggers abren un arco que pendula entre la adecuación a los preceptos que definieron la buena madre en el siglo XX y la reacción crítica ante tales exigencias sociales con la reivindicación de quienes se asumen "las malas madres" (Barbosa y Crespo Cabillo, 2015).

Respecto a que esos espacios sean un lugar de encuentro para acceder a lazos de sostén emocional y amistad, su función de sociabilidad resulta significativa para las mujeres - generalmente de sectores medios, residencia urbana y familia nuclearen virtud, como observa Carolina De Olmo (2013), de la soledad en la que se cumplen las tareas de maternaje en el marco del orden capitalista neoliberal.

Las notas periodísticas sobre las cuentas de los medios sociales sobre maternidad caracterizan estos espacios como de acceso a una experiencia colectiva que, aunque virtual, son valorables por constituir una comunidad en la cual las madres 
identifican sus emociones en relatos de pares, obtienen e intercambian información y encuentran referencias que mesuran el modelo inalcanzable e impracticable de la crianza y la maternidad ideales.

Por ejemplo, en un artículo ${ }^{8}$ sobre blogs de maternidad, la revista Elle destaca la función de sociabilidad con la siguiente presentación: "Con humor, realismo y ternura, estos blogs arman comunidad, brindan contención y funcionan de nexo para que las madres se sientan menos solas en su titánica tarea”. En los testimonios de las bloggers que incluye la publicación, la referencia a la soledad como dimensión del ejercicio materno y de las redes como sitios de encuentro y construcción de comunidades se reitera:

Las madres están muy solas: el afuera es cero empático. Todos opinan y nadie acompaña. Entonces la gente encuentra en las redes un espacio horizontal para hablar de igual a igual y no sentirse sola.

La comunidad es todo, es la razón de ser de la cuenta y es lo que nos demuestra día a día que el contenido que generamos sirve para algo. No es algo unidireccional, y en la mayoría de los casos es más lo que nos da la comunidad a nosotras que al revés. Nos apoyan, aconsejan, nos dan valor para seguir enfrentando los desafíos que aparecen en la maternidad. El vínculo es muy real (S/A, 2018, online).

No obstante, a pesar de referir que la valoración de estos espacios reside en su capacidad de configurar lazos, la naturalización del carácter individual del cuidado infantil (con su concomitante feminización y su preponderante atribución a las madres como el sujeto responsable preferible) es una dimensión que no aparece problematizada en los medios masivos. O que, al señalarse, se presenta -como lo hace en una nota ${ }^{9}$ el sitio periodístico Infobae- en tanto consecuencia de la participación de las mujeres en la esfera del trabajo productivo:

[...] luego de un tiempo en el que la mujer logró su inserción en el campo laboral, y madres, tías y amigas dejaron de estar "disponibles" para criar en grupo, lo natural vuelve a tomar su curso y las tribus de crianza y blogs de maternidad parecen ser el espacio donde "maternar" se hace más fácil (Chavez, 2018, online).

8 S/A. Mami blogs: conocé las nuevas influencers. Op.Cit.

9 Chavez, Valeria (9/6/2018). Los blogs de maternidad como nuevas tribus de crianza, y 5 influencers que se convirtieron en el espejo donde se miran las madres hoy. Infobae [online]. Disponible en: https://www.infobae.com/tendencias/2018/o6/o9/los-blogs-de-maternidad-como-nuevas-tribusde-crianza-y-5-influencers-que-se-convirtieron-en-el-espejo-donde-se-miran-las-madres-hoy/ (consulta: 24/11/2020). 
Las mujeres crían solas, dice Infobae, porque se desplazaron de la esfera reproductiva a la productiva y ya no están disponibles para tejer redes de ayuda entre ellas. El enfoque de la nota concibe como "natural" que la crianza esté a cargo de mujeres, y que estas se contengan y ayuden mutuamente en las tareas de cuidado, y subraya entonces que los medios sociales reconstituyen en el paisaje digital la disponibilidad de lazos de acompañamiento para ejercer el cuidado materno.

De este modo, la naturalización del confinamiento histórico de las mujeres a las tareas reproductivas como una responsabilidad exclusiva de las madres se entrama en un nuevo emplazamiento que Gina Masullo Chen (2013) apunta como la domesticidad digital. Una domesticidad que se configura como espacio colectivo, pero para sobrellevar de mejor modo el carácter individual y feminizado de la crianza (y de las tareas requeridas para cubrir las necesidades de quienes integran una familia) que continúa incuestionado.

Respecto a la reproducción social de la naturalización y asignación feminizada del cuidado, la institucionalización del trabajo doméstico como una tarea propia de las mujeres es el engranaje clave de la división sexual del trabajo del patriarcado capitalista (Mies, 2019). Al analizar el proceso de construcción del ama de casa y del trabajo doméstico en Inglaterra, Silvia Federici (2018) recorre el trayecto histórico en el que, con reformas en la regulación laboral orientadas a beneficiar a los trabajadores varones, se configuró una nueva moralidad sobre el rol social de las mujeres. La delimitación como gestoras de los cuidados del hogar cimentó el desplazamiento de las mujeres del trabajo asalariado al no asalariado dotando reconocimiento social a las que se consagraban exclusivamente a las tareas reproductivas. Al mismo tiempo, en ese proceso se erigió la respetabilidad masculina en la capacidad individual de solventar la vida familiar.

En la economía simbólica, las relaciones sociales de explotación y subordinación de las madre-esposas a los maridos quedaron opacadas bajo la mistificación del amor y por la concepción del trabajo reproductivo como inherente a la "naturaleza" de las mujeres en función de sus capacidades en la reproducción biológica de la vida humana (Mies, 2019). La creación de la familia nuclear a partir del "patriarcado del salario", es decir, de la dependencia de las mujeres del salario de los trabajadores varones ${ }^{10}$ (Federici, 2018, p. 13) implicó una jerarquización de las relaciones socio-sexuales en la cual las mujeres fueron disciplinadas para cumplir funciones excluyentes (aunque desvalorizadas) para la acumulación de capital.

Así, la pregnancia de comprender la feminización del cuidado y las labores domésticas como un proceso natural y carente de aportes al funcionamiento del

10 El proceso de institucionalización del "ama de casa” situado, fundamentalmente, en el siglo XIX refiere a condiciones de organización socio-económica centradas en los modos de vida de la burguesía y las clases medias, en primera instancia; extendidos luego también, como analiza Federici (2018) a las clases trabajadoras. En cambio, según explica Davis (2005), las mujeres negras transitaron una experiencia de explotación (y de lucha) diferente, en tanto no fueron objeto de la exigencia social a asumir una dedicación total al trabajo doméstico. 
orden socio-económico, como una determinación biológica que responde a instintos innatos, a una esencia que atraviesa la feminidad cuya expresión emocional es la entrega desinteresada de y por amor, nutrió ideológicamente la glorificación de la familia y de la maternidad (Badinter, 1980; Federici, 2018, p. 32-33). Volviendo a los blogs e instagrams de maternidad otro aspecto que las notas periodísticas ponderan es que sus autoras articulan con eficiencia, y algún sobresalto menor, el trabajo reproductivo con el productivo:

Cocinan, hacen arreglos en la casa, llevan y traen niños a sus actividades, y además trabajan fuera del hogar, tienen reuniones en el colegio, lidian con clientes y llegan tarde a turnos médicos: son mamás full-time (Chavez, 2018, online)" .

La incorporación masiva de las mujeres (y por lo tanto de las madres) al mundo del trabajo productivo en el siglo XX no modificó la división sexual del trabajo, pivote que sostiene -como se mencionó- el patriarcado capitalista. Por eso mismo, para conciliar la participación en la esfera productiva y cumplir las exigencias de la reproductiva, las mujeres se han constituido en un nuevo sujeto social: las malabaristas; o, en términos de Eleonor Faur, "Mujeres-todo-terreno que sustentan la estructura económica, social y familiar sobre su esfuerzo cotidiano de gestión y ejecución de múltiples tareas presuntamente coordinadas" (2014, p. 253).

Si las bloggers/instamums a las que alude el periodismo son "mamás full-time" que realizan las piruetas y las destrezas requeridas para combinar los eslabones de la vida profesional y la familiar, el modelo contemporáneo que presenta de modo positivo tal capacidad se resume en la idea de las madres emprendedoras: "que, para cuidar a sus hijos, decidieron resignar un sueldo fijo; madres que no podían dejar de trabajar, pero que encontraron el equilibrio para mantener la economía y la vida familiar" (Andahazi, 2015, online) ${ }^{12}$.

\section{La Mompreneur}

El lazo entre maternidad y emprendedorismo se enuncia con un acrónimo anglosajón: la mompreneur, es decir la madre emprendedora. Un modelo presentado como el de las madres que gestionan con éxito un proyecto laboral impulsado por ellas mismas y que, al mismo tiempo, ejercen del modo considerado socialmente apropiado las tareas de cuidado de los hijos y del hogar:

11 Chavez, Valeria. Op.Cit.

12 Andahazi, Laura (15/10/2015): La maternidad, una excusa para emprender. El Cronista [online]. Disponible en https://www.cronista.com/pyme/La-maternidad-una-excusa-paraemprender-20151015-0002.html?utm_source=ecc_nota\&utm_medium=cms\&utm_campaign=refresh (consulta: 3/12/2020). 
Hay un rasgo distintivo y relevante en todas estas mujeres, no disocian la vida familiar de sus proyectos de trabajo, no los separan en dimensiones diferentes, ni ocultan una cuando hablan de la otra, como solía pasar, sino que las integran y muchas veces hasta generan sinergias, ya sea simbólicamente, como el apoyo y la energía familiar, hasta en muy concretas y originales ideas de negocio (Montero, 2017, online) ${ }^{13}$.

El rasgo que posibilita la capacidad de proyectar y concretar una actividad lucrativa propia gestionada a través de los medios sociales, dicen los discursos periodísticos, es justamente ser madre: "La maternidad, ese impulso para emprender" (Cattaneo, 2016, online) ${ }^{14}$, "La maternidad, una inspiración para emprender"(Lavate, 2018, online) ${ }^{15}$, "La maternidad, una excusa para emprender"(Andahazi, 2015, online $)^{16}$, titulan distintas notas. Sin embargo, los artículos periodísticos no exponen argumentos sobre por qué la maternidad estimula o impulsa esa capacidad sino que ofrecen relatos que detallan experiencias diferentes de madres que tienen actividades productivas a partir de iniciativas comerciales, en gran medida asociadas a la maternidad (como los blogs y cuentas en las redes sociales sobre embarazo y crianza u otro tipo de productos como indumentaria, mobiliario o alimentación infantil).

En los medios sociales, las cuentas de las usuarias aludidas por el periodismo también acuden a la clasificación de "mamá emprendedora" y alientan a la asunción de este modelo. Por ejemplo, Fabiana Jafif, del blog De Madre a Madre, sugiere que las madres deben dejar "de usar a maternidad como excusa" para "usarla como ¡MOTIVACIÓN! Hay que empezar a ganarles a lo que yo llamo los «No puedo de la maternidad»" (Jafif, 2019, online) ${ }^{17}$. Las claves que propone Jafif son (para la organización del emprendimiento) una administración minuciosa del tiempo, la delegación de tareas y (para las tareas de crianza) la solicitud de ayuda. Sobre esto último, afirma que aunque "las mujeres tengamos el don del multitasking" no hay modo de resolver todo lo relativo al cuidado infantil y sugiere hablar con la pareja y dividir las responsabilidades. Además de administrar un blog con reflexiones sobre la experiencia materna, consejos para el embarazo y la crianza y de promoción de eventos y productos, Jafif organiza charlas y cursos para ser una madre emprendedora y profesionalizarse en la gestión de herramientas digitales.

13 Montero, Laura (9/5/2017): Mompreneurs: la palabra que describe una nueva realidad del mundo de las mujeres. Coca Cola Journey [online]. Disponible en: https://journey.coca-cola.com/historias/ mompreneurs--la-palabra-que-describe-una-nueva-realidad-del-mund (consulta: 4/12/2020).

14 Cattaneo, Carolina (5/10/2016): La maternidad, ese impulso para emprender. Sophia [online]. Disponible en https://www.sophiaonline.com.ar/maternidad-emprender/ (consulta:3/12/2020).

15 Lavate, Marianela (18/7/2018): La maternidad, una inspiración para emprender. El Cronista [online]. Disponible en https://www.cronista.com/pyme/negocios/La-maternidad-una-inspiracion-paraemprender-20180718-0002.html (consulta: 3/12/2020).

16 Andahazi, Laura. Op.Cit.

17Jafif, Fabiana (23/8/2019): Balance. Cómo combinaremprendimientoy maternidad. La Nación [online]. Disponible en https://www.lanacion.com.ar/lifestyle/balance-como-combinar-emprendimientomaternidad-nid2280370 (consulta: 4/12/2020). 
Beta Suarez, propietaria del blog Mujer, Madre y Argentina, es una de las madres bloggers más referidas por las notas periodísticas por su larga trayectoria en la blogosfera materna argentina. En sus espacios digitales, abarrotados de auspiciantes, escribe sobre la maternidad y los vínculos de pareja, la amistad, las mujeres, entre otros tópicos, a partir de sus propias vivencias. Profesional de la comunicación, también ofrece servicios de capacitación y participa en charlas sobre emprendedorismo. Galardonada por la revista OhLaLa como una de las emprendedoras "más inspiradoras del año", afirma que en los primeros años "la maternidad tiene una gran parte de postergación", pero sostiene que no hay que renunciar a los afanes ya que "si los hijos son lo más lindo que nos pasó en la vida, no está bueno que los usemos de excusa para posponer nuestros sueños" (Vissanasi y Simond, 2019, online) ${ }^{18}$.

En el caso del blog Mamá Emprende, creado por la publicista Ale Leguizamón, el objetivo es específicamente capacitar y vender servicios a las madres que quieren armar un emprendimiento "en todas las áreas que involucran la creación de un negocio" como el nombre de la marca ("namming”), el diseño de producción, la producción, precios, fotografía, marketing, tienda digital, mailing y el contenido para redes sociales ${ }^{19}$. Las entradas del blog se orientan a responder preguntas para la gestión de los espacios digitales, tales como: “¿Por qué pierdo seguidores en Instagram?” o “Cómo escribir la BIO de Instagram?”.

Observamos que un rasgo del modelo mompreneur es que se acomoda al molde subjetivo del neoliberalismo, apoyado en un discurso en el cual el esfuerzo y la voluntad, la proactividad, la capacitación permanente, la gestión del sí mismo como una empresa que hay que administrar con eficacia expresa valores modelizadores de las prácticas. Para el que no hay imposibilidades individuales y para el que las estructuras sociales de desigualdad (de género, de raza, de clase) son "excusas" que se sortean con organización.

La perspectiva neoliberal del modelo mompreneur se combina, a la vez, con lo que Sharon Hays llama la "ideología de la maternidad intensiva" (1998, p. 28-31). La maternidad intensiva implica una forma de interpelación que significa a las madres como responsables del cuidado infantil, exige un modo de maternar que acopie y ponga en práctica las consideraciones de los saberes expertos, determina la disponibilidad de considerables recursos económicos y se organiza en torno a una perspectiva moral que niega la valuación del trabajo reproductivo. Hays observa que las madres que se desempeñan en tareas remuneradas lidian con una trama contradictoria entre la ideología de la maternidad intensiva y la ideología de mercado que permea a los sujetos en el orden capitalista (guiada por el interés propio y el individualismo). Y señala que ésta última resulta incoherente con la configuración subjetiva de entrega desinteresada que se determina para

18 Vissanasi, Agustina y Simond, Soledad (11/10/2019): Beta Suarez: Los hijos no pueden ser una excusa para lo que no hacemos. La Nación [online]. Disponible en: https://www.lanacion.com.ar/lifestyle/ beta-suarez-los-hijos-no-pueden-ser-nid2293049 (consulta:3/12/2020).

19 S/A. Te ayudamos a emprender. Mamá Emprende. Disponible en: https://www.mamaemprende. com.ar/ (consulta: 5/12/2019). 
las madres. Es entre esas lógicas contradictorias y en tensión que transcurren las experiencias de las madres que se desempeñan simultáneamente en la esfera productiva y en la reproductiva.

Además de las bloggers mencionadas, muchas otras madres que producen contenidos para medios sociales, cuyos nombres se divulgan en notas periodísticas, aplican a la categoría mompreneur: Bernardita Siutti es Mami Albañil y realiza tutoriales para enseñar tareas vinculadas a la albañilería; Feli de la Garma es Mamá Sobre Ruedas y produce videos cómicos sobre la cotidianidad de una madre que -para resolver la crianza- pasa gran parte de su vida manejando su automóvil; Debbie Maniowicz es actualmente en las redes Upalala y escribe sobre temáticas como lactancia, crianza y partos respetuosos; Ximena Bruno gestiona It Baby Eat y aconseja sobre alimentación. Estas mujeres, que comparten su posición social de profesionales de sectores privilegiados y la labor como instamums, expresan que lo que las llevó a buscar una actividad productiva como la creación de contenidos digitales fue la decisión de no delegar la crianza para priorizar a sus hijas e hijos ${ }^{20}$. Por ejemplo, Ximena Bruno cuenta:

Antes estudiaba ingeniería civil y trabajaba en Hidráulica. Mi último trabajo fue en la Reserva Natural Costanera Sur dirigiendo el proyecto de restauración de las lagunas. Un trabajo espectacular que involucró muchísimo el cuidado del medio ambiente, áreas que me fascinan. Pero ahora siendo mamá mis tiempos y prioridades son otros, no podría estar todo el día afuera de casa trabajando y después cursando y estudiando (Chavez, 2018, online) $)^{21}$.

Por otra parte, las llamadas mompreneurs e influencers que personalizan las notas periodísticas señalan, al mismo tiempo, la pretensión de desidealizar la maternidad para que las madres no persigan encarnar exigencias inalcanzables que producen insatisfacción y culpa; incluso refieren que sus contenidos buscan habilitar que las mujeres se piensen capaces de tareas que son socialmente dominio de lo masculino:

Principalmente desdramatizar. Hace que todo sea más liviano. Saber que no sos la peor mamá de mundo, ni tampoco la mejor [Feli de la Garma](Chavez, 2018, online) ${ }^{22}$.

Yo hablo más de madres que de hijos. De cómo la maternidad atraviesa todo el resto de nuestras facetas sin la obligación de tener que anularlas. De cómo los hijos son lo más importante

\footnotetext{
20 Chavez, Valeria. Op.Cit.

21 Chavez, Valeria. Op.Cit.

22 Chavez, Valeria. Op.Cit.
} 
de nuestras vidas pero no son lo único. De cómo sacarnos de encima tanta definición forzada de la maternidad para construir la propia. Desde el blog promovemos aquello de que no hay dos maternidades iguales y que la riqueza es justamente encontrarnos en esa diversidad [Beta Suarez] (S/A, 2018, online $)^{23}$.

No es un blog de crianza, no doy consejos porque creo que cada madre hace lo que puede. Pero siento que les dejo un mensaje súper importantea mis hijas. Que vean que una mujer puede hacer los mismos arreglos que antes los hacía el padre, que se puedan valer por ellas mismas sin depender de un varón para cambiar un canilla [Bernardita Siutti] (S/A, 2018, online) ${ }^{24}$.

En tal sentido, es claro que estas propuestas se inscriben en un clima de época en el que las identidades consideradas inmutables en otro tiempo están siendo interrogadas y transformadas. Y que -como otros repertorios de la maternidad- señalan que el ideal de la madre tradicional e, incluso, la ideología de la maternidad intensiva son verosímiles en crisis. Estos matices dan cuenta de que las narrativas actuales sobre la maternidad expresan, simultáneamente, operaciones de perturbación y reproducción simbólica en torno a las formas en que las madres son significadas dentro y con los límites de los márgenes culturales y materiales del sistema patriarcal.

Ahora bien, en esa tensión las influencers insisten -con las experiencias de vida que encarnan- en la posible conciliación con éxito y eficiencia de las tareas de las esferas productiva y reproductiva. Es decir, reconocen en sus relatos el antagonismo implicado en tal articulación como una trama de dificultades pero representan un modelo en el que, en definitiva, el estado de armonía entre ambas esferas -como un horizonte alcanzado y alcanzable- es el que prevalece.

\section{Reflexiones Finales}

Este acercamiento a un recorte del repertorio que, provisoriamente, puede identificarse como digital permite unas últimas reflexiones sobre algunas dimensiones de este universo. En primer lugar, señalar -como se dijo- que el espacio narrativo de la maternidad en los medios sociales emerge centrado en la experiencia desde las voces de las madres (lo que marca una distancia respecto a relatos en el que las voces son las del saber experto). Por eso mismo, un abordaje profundo del fenómeno de los sitios de red social y producción de contenidos sobre maternidad en Argentina implicaría una clasificación que agrupe la variedad de líneas que lo componen, dentro de las cuales las blogger-insta-mompreneurs son solamente uno

23 S/A. Mami blogs: conocé las nuevas influencers. Op.Cit.

24 S/A. Mami blogs: conocé las nuevas influencers. Op.Cit. 
de los relatos a observar. La gran cantidad de cuentas que es posible advertir al ingresar palabras-clave en buscadores de internet o de los propios medios sociales requiere señalar, por ejemplo, que es un fenómeno teñido también por el proceso de creciente participación de las madres en la organización de demandas y acciones de los feminismos.

En segundo lugar, en relación a lo explorado para este trabajo sobre ideas que circulan en medios masivos en torno a las bloggers o instamums que son destacadas por su gran cantidad de seguidoras y/o por su trayectoria en la blogosfera materna, nos interesa pensar nuevamente en torno a la evaluación de este tipo de espacios como constituyentes de comunidad y facilitadores de encuentros colectivos. Dado que es un rasgo señalado por las notas periodísticas y una cualidad que encuentran quienes han investigado en torno a la blogosfera materna al recuperar lo enunciado por quienes realizan y/o participan en ese lugar. Y que las influencers de la maternidad también aseguran que, con la producción de contenidos, se posibilita un nexo que construye ciertos lazos entre madres a través del sentido de pertenencia a una comunidad digital.

Podría interpretarse que el carácter colectivo de la maternidad en estos espacios se centra en sobrellevar las imposiciones sociales y transitar las exigencias con algunas críticas al imaginario de la madre ideal que, en efecto, distiende las insatisfacciones. Pero esa colectividad digital, en el caso que observamos acá, se mantiene acomodada al escenario de subordinación cuando, aunque aluda a la soledad y los juicios que de modo permanente padecen las madres y valore los intercambios de información y la manifestación de emociones como una forma de componer grupalidad, lo que no se problematiza es su carácter exclusivamente individual.

Ese carácter individual de la maternidad se reproduce en las narrativas que proponen testimonios de experiencias que, permeadas por la feminización de las tareas de cuidados, exponen casos o modelos exitosos del ejercicio materno y de conciliación entre el trabajo productivo y reproductivo.

Las estrategias que se divulgan, ofrecen modos en los que la resolución compromete la capacidad y habilidad de las madres a partir de una operación retorica central de desdramatización o atenuación del desborde, de los malabares y de la insatisfacción; por ser un estado compartido y reconocido que, además, ahora puede ser enunciado públicamente.

El siguiente movimiento de la operación de desdramatización es una modalidad declamativa que sostiene "las madres hacemos lo que podemos" mientras, sin embargo, los modelos influencers hacen todo lo que se espera de las madres contemporáneas: priorizan la crianza desde una maternidad individual y sostienen emprendimientos lucrativos exitosos.

En resumen, la colectividad constituida -bajo estos términos- reubica a las madres en el espacio de la domesticidad como esfera feminizada de responsabilidad exclusivamente individual. Una domesticidad que se vuelve más elástica, que amplía sus fronteras para incorporar mompreneurs y que suma una dimensión digital en 
la que -con algunas pinceladas críticas- se abona el emplazamiento de las madres asignado por el sistema capitalista patriarcal.

\section{Referencias}

ANGENOT, Marc. El discurso social. Buenos Aires: Siglo XXI, 2010.

ARFUCH, Leonor. El espacio biográfico. Buenos Aires: Fondo de Cultura Económica, 2007.

BADINTER, Elisabeth. ¿Existe el amor maternal? Historia del amor maternal. Siglos XVII al XX. Barcelona: Ediciones Paidós Ibérica, 1980.

DAVIS, Angela. Mujeres, raza y clase. Madrid: Akal, 2005.

DE OLMO, Carolina. ¿Dónde está mi tribu? Maternidad y crianza en una sociedad individualista. Buenos Aires: Capital Intelectual, 2013.

FAUR, Eleonor. El cuidado infantil en el siglo XXI. Mujeres malabaristas en una sociedad desigual. Buenos Aires: Siglo Veintiuno Editores, 2014.

FEDERICI, Silvia. El patriarcado del salario. Buenos Aires: Tinta Limón, 2018.

HAYS, Sharon. Las contradicciones culturales de la maternidad. Barcelona: Paidós, 1998.

MASULLO CHEN, Gina. Don't Call Me That: A Techno-Feminist Critique of the Term Mommy Blogger. Mass Communication and Society, vol. 16, n. 4, 2018, p. 510-532.

MIES, María. Patriarcado y acumulación a escala mundial. Madrid: Traficante de Sueños, 2019.

ORTON-JOHNSON, Kate. Mummy Blogs and Representations of Motherhood: 'Bad Mummies' and Their Readers. Social Media + Society, abr./jun. 2017, p. 1-10.

RICHARD, Nelly. La crítica feminista como modelo de crítica cultural. Debate feminista, vol. 40, 2009, p. 75-85.

SIBILIA, Paula. La intimidad como espectáculo. Buenos Aires: Fondo de Cultura Económica, 2008. 
THOMPSON, John. La comunicación masiva y la cultura moderna. Contribución a una teoría crítica de la ideología. Revista Versión. Estudios de comunicación y política, n. 1, 1991, p. 43-76.

VAN DIJCK, Jose. La cultura de la conectividad. Una historia crítica de las redes sociales. Buenos Aires: Siglo Veintiuno Editores, 2016.

VISA BARBOSA, Mariona y CRESPO CABILLO, Cira. El papel de la blogosfera en la construcción social de la maternidad: de la Virgen María a las \#malasmadres. Revista de Comunicación de la SEECI, ano XIX, n. 37, jul. 2015, p. 299-331.

Recebido em 21/03/2021.

Aceito em 05/06/2021. 